\title{
Erratum to: Effect of augmentation techniques on the failure of pedicle screws under cranio-caudal cyclic loading
}

\author{
Richard Bostelmann ${ }^{1} \cdot$ Alexander Keiler ${ }^{2} \cdot$ Hans Jakob Steiger ${ }^{1} \cdot$ \\ Armin Scholz $^{3} \cdot$ Jan Frederick Cornelius $^{1}$ - Werner Schmoelz ${ }^{2}$
}

Published online: 13 May 2015

(C) Springer-Verlag Berlin Heidelberg 2015

\section{Erratum to: Eur Spine J \\ DOI 10.1007/s00586-015-3904-3}

Despite thorough review of the manuscript an error has occurred. One of the figures has been interposed somehow. Now the correct Fig. 4 is presented. It is illustrating that the control screwing technique exhibited significant lower load cycles to failure compared to all other groups. The boxplots are showing the median, the 25 th and the 75 th quartile of the load cycles to failure for the four groups. Statistical significant differences to the control group are marked with an asterisk.

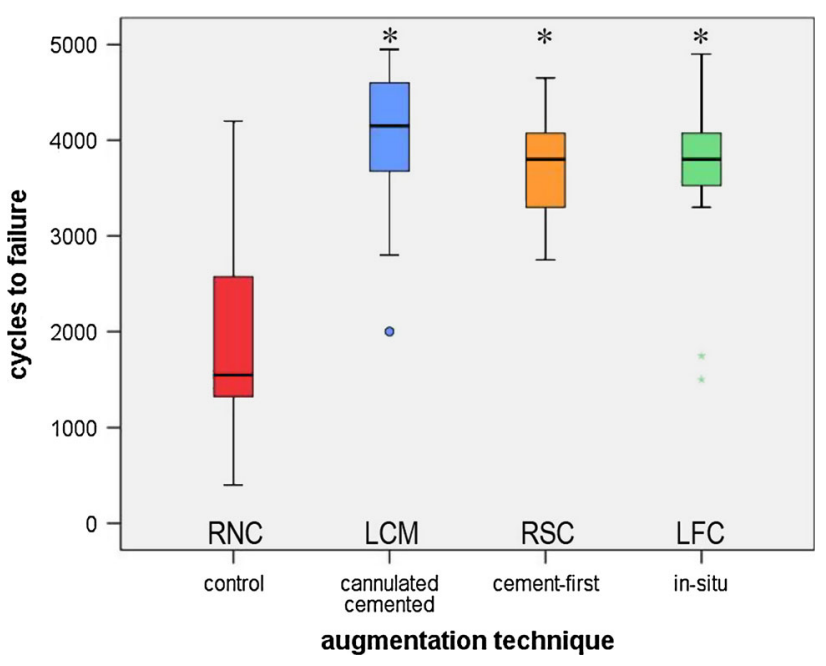

Fig. 4 The control screwing technique exhibited significant lower load cycles to failure compared to all other groups. The boxplots are showing the median, the 25 th and the 75 th quartile of the load cycles to failure for the four groups. Statistical significant differences to the control group are marked with an asterisk
The online version of the original article can be found under doi:10.1007/s00586-015-3904-3.

Richard Bostelmann

richard.bostelmann@med.uni-duesseldorf.de

1 Department of Neurosurgery, University Hospital of Duesseldorf, Moorenstrasse 5, 40225 Düsseldorf, Germany

2 Department of Trauma Surgery, Medical University Innsbruck, Innsbruck, Austria

3 Department of Trauma Surgery, University Hospital of Duesseldorf, Düsseldorf, Germany 